\title{
STUDY OF URINARY ISOLATES WITH REFERENCE TO EXTENDED SPECTRUM BETA LACTAMASES DETECTION AND ANTIBIOGRAM
}

Abhijit Awari, Sunita Nighute, Maria Khatoon

1. Associate Professor. Department of Microbiology, Kesarsal Medical College and Hospital, Ahmedabad.

2. Assistant Professor. Department of Physiology, Kesarsal Medical College and Hospital, Ahmedabad.

3. Assistant Professor. Department of Physiology, People's Medical College and ResearchCentre, Bhopal.

\section{CORRESPONDING AUTHOR}

Abhijit Awari,

Associate Professor, Dept of microbiology,

People's medical college \& RC,

Bhanpur, Bhopal, MP.

E-mail: abhijit.awari@yahoo.com

Ph: 00917898726364,00917898726341

ABSTRACT: BACKGROUND: Extended spectrum beta lactamases continue to be major problem in clinical setups world over, conferring resistance to extended spectrum cephalosporins and are associated with significant morbidity and mortality. Urinary tract infections (UTIs) are one of the most common infectious diseases encountered in the clinical practice. Extended spectrum beta lactamases (ESBLs) production in gram negative bacteria, have emerged as a major problem in hospitalized as well as community based patients. ESBLs producing bacteria may not be detected by routine disc diffusion susceptibility test, leading to inappropriate use of antibiotics and treatment failure. The objective of this study was to determine the resistance patterns of the micro-organisms isolated from cases of UTI and to detect ESBLs production in gram negative bacteria. METHODS: Urinary isolates from symptomatic UTI cases (both in patients and out patients) attending the, Kesarsal Medical College and Hospital Ahmadabad were identified by conventional methods. Antimicrobial susceptibility testing was performed by Kirby Bauer's disc diffusion method gram negative isolates resistant to third generation cephalosporins were tested for ESBL production by two methods. RESULTS: Number of urinary isolates from patients with symptomatic UTI was 350 over a study period of one year. E.coli was the predominant isolate (57.7\%) both in IPD and OPD patients. A total of 171 gram negative isolates resistant to third generation cephalosporins were tested for ESBL production by two methods- Modified Double Disc Synergy Test (CLSI) Phenotypic Confirmatory Test (PCT). ESBL production was seen in $36(21.05 \%)$ isolates. Maximum ESBL production was seen in $K$. pneumoniae (22.41\%) isolates followed by E.coli (13.26\%). CONCLUSION: This study showed E.coli to be the predominant urinary pathogen isolated from UTI cases. Overall incidence of ESBL producing microorganisms was $21.05 \%$.

KEYWORDS: Urinary tract infections,, antimicrobial resistance, ESBLs.

Me SH TERM - ESBL, Antibiogram.

INTRODUCTION: The extended spectrum beta lactamases are typically plasmid mediated enzymes that hydrolyze penicillin's, third generation cephalosporins and aztreonam. ${ }^{1}$ Urinary tract infections (UTIs) are one of the most common infectious diseases encountered in the clinical practice, mainly associated with different members of the family Enterobacteriaceae 2.Bacteria responsible for UTI, often originate from the fecal and perineal flora ${ }^{3,4}$. Antibiotics are 
usually given empirically before the laboratory results of urine culture and sensitivity are available 5 .

Resistant bacteria are emerging world wide as a threat to the favorable outcome of common infections in community and hospital settings. $\beta$ lactamases production by several gram negative and gram positive organisms is perhaps one of the most important single mechanism of resistance to penicillins and cephalosporins 6 .

Extensive use of third generation cephalosporins has contributed to the evolution of extended spectrum beta lactamases (ESBLs). These plasmid mediated groups of enzymes are the product of point mutations at the active site of TEM, SHV, and OXA enzymes 7 .

To ensure appropriate therapy, current knowledge of the organisms that cause UTI and their antibiotic susceptibility pattern is mandatory ${ }^{8}$. Since patterns of antibiotic resistance in a wide range of pathogenic organisms may vary over short periods, depending on the site of isolation and on different environments; periodic evaluation of antibacterial activity is needed to update this information 9,10,11.

This study was conducted with an aim to determine the resistance patterns of the microorganisms isolated from suspected cases of UTI and to detect ESBLs production in gram negative isolates.

MATERIALS AND METHODS: A prospective study of 1084 urine samples from symptomatic UTI cases (both IPD and OPD) received in the Department of Microbiology, Kesarsal Medical College \& Hospital Ahmadabad, was carried out over a period of one year .Majority of the samples were Clean catch midstream urine sample (CCMSU) and others included aseptically collected catheterized urine sample and suprapubic aspirates. Urine samples were microscopically studied by wet mount preparation and gram staining,(C16) inoculated on 5\% sheep blood agar and Mac-Conkey's agar and incubated at $37^{\circ} \mathrm{C}$ for 24 hours.

Semi quantitative urine culture using a calibrated loop was done on blood agar and MacConkey's agar plates. Following Kass criteria, significant monomicrobic bacteriuria was defined as culture of a single bacterial species from the urine samples at a concentration of $>10^{5}$ $\mathrm{CFU} / \mathrm{ml} \mathrm{12,13}$ Only a single positive culture per patient was included in the study. Microorganisms were identified by standard biochemical procedures ${ }^{14,15}$.

Antibiogram of the isolates was done by Kirby Bauer's disc diffusion method using antibiotic discs from Himedia laboratories.

Antibiotics used for gram negative bacteria were Ampicillin (100 mcg), cephalexin (30 $\mathrm{mcg}$ ), cefotaxime (30 mcg), ceftazidime (30 mcg), amikacin (30 mcg), nitrofurantoin (300 mcg), nalidixic acid (30 mcg), norfloxacin (10 mcg), co-trimoxazole $(25 \mathrm{mcg})$, and gentamicin (10 $\mathrm{mcg})$. For Pseudomonas aeruginosa, piperacillin (100 mcg) was also used.

Gram negative isolates having zone size of $<22 \mathrm{~mm}$ for ceftazidime (standard disc diffusion technique) were selected as suspicious for ESBL production as recommended by CLSI guidelines. These potential ESBL producing strains were further tested by two methods.

1) Modified Double disc synergy test (DDST) ${ }^{16}$ : Lawn culture of test strain on Mueller Hinton agar (Himedia, Mumbai) was exposed to discs of cefotaxime (30 mcg), ceftazidime (30 mcg), and the disc of amoxiclav (augmentin) (20ug amoxicillin / 10ug clavulanic acid). The cefotaxime and ceftazidime disc were placed $16 \mathrm{~mm}$ center to center from amoxiclav disc. Plate was incubated at $37^{\circ} \mathrm{C}$ overnight. The test isolate 
was considered to produce ESBL, if the zone size around the cefotaxime and ceftazidime disc increased towards the augmentin disc.

2) CLSI phenotypic confirmatory test (PCT) ${ }^{17}$ :Lawn culture of test isolates was done on Muller Hinton agar. Antibiotic used were Ceftazidime(30 mcg) and combination of ceftazidime-clavulanic acid $(30 \mathrm{mcg})$. Discs were placed opposite to each other in Muller Hinton agar plate and incubated overnight at $37^{\circ} \mathrm{C}$. Next day zone of inhibition around ceftazidime and ceftazidime clavulanic acid was measured. If zone of inhibition around ceftazidime-clavulanic acid is increased by more than $5 \mathrm{~mm}$ than that of ceftazidime disc alone. It is confirmed that isolate was ESBL producer.

E.coli ATCC 25922 was used as ESBL negative control and Klebsiella pneumoniae ATCC 700603 was used as ESBL positive control.

RESULTS: A total of 350 uropathogens were isolated from symptomatic UTI patients. E.coli was the predominant isolate $(57.7 \%)$ followed by K.pneumoniae $(28.3 \%)$. Other isolates are shown in( Table No. 1.)

UTI was more common in female patients as compared to males (Table No.2). The commonest age group affected in males was 41-50 years and in female was 21-30 years. Antibiotic resistance pattern showed E.coli to be maximum resistant to amino penicillin followed by cephalexin. Most effective antibiotic against E.coli was nitrofurantoin. For K.pneumoniae, gentamicin, amikacin and cotrimoxazole were found to be effective (Table No.3).

E.coli $(\mathrm{n}=98)$, K.pneumoniae $(\mathrm{n}=58)$ and other gram negative bacilli resistant to third generation cephalosporins (cefotaxime and ceftazidime) were tested for ESBL production by two methods. ESBL production was seen in 36 (21.05\%) isolates out of a total of 171 tested. Maximum ESBL production was seen in K.pneumoniae isolated from IPD patients $(22.41 \%)$ followed by E.coli (13.26\%). PCT was found to be better than modified DDST for detection of ESBL production (Table No. 4).

DISCUSSION: Despite the widespread availability of antibiotics, UTI remains the most common bacterial infection in the human population.

In the present study E.coli was the predominant isolate followed by K.pneumoniae. This tallies with the studies of other workers like Varma $\mathrm{N}$ et al ${ }^{18} \mathrm{Gupta} \mathrm{V}$ et al ${ }^{19}$. Our findings, however contrast with the study of Bajaj et al ${ }^{20}$ where Klebsiella species predominated E.coli. Both host and bacterial factors have been associated with the pathogenesis of UTI. Uropathogenic strains of E.coli are believed to display a variety of virulence properties that help them to colonize the host mucosal surfaces and circumvent host defenses to allow invasion of normally sterile urinary tract 21,22 .

Female patients presenting with symptoms of UTI were more as compared to male patients. In general, rates of UTIs are higher among women than among men, with cystitis being the most prevalent UTI. By routine disc diffusion susceptibility tests, 166 out of $341(48.7 \%)$ gram negative isolates showed resistance to cefotaxime whereas 164 (48.1\%) were resistant to ceftazidime. A total of 171 gram negative bacteria resistant to third generation cephalosporins were tested for ESBL production by two methods. ESBL production was detected in $27(15.8 \%)$ isolates by modified DDST whereas; additional 09 ESBL producers were detected by CLSI PCT (21.05\%). Various factors like precise placement of the discs, correct storage of the clavulanate containing disc and performance of appropriate control tests are critical to the sensitivity of $\mathrm{m}$ DDST ${ }^{23}$. In comparison to this, PCT is simple, cost effective and easy test to 
perform; therefore it can be used as a routine test for ESBL detection. Maximum incidence of ESBL production was seen in K. pneumoniae (22.41\%) isolates followed by E.coli $(13.26 \%)$ from indoor patients. High prevalence rate of ESBL producing strains have been reported in Klebsiella spp by Gupta $\mathrm{V}$ et al ${ }^{19}$ and Akata $\mathrm{F}$ et al 24

Iqbal $\mathrm{M}$ et al ${ }^{25}$ have reported ESBL production in E.coli ranging between 21 to $34 \%$. One of isolate of Ps. a.eruginosa was found to be ESBL producer in our study Acinitobater species \& proteus mirabilis were negative for ESBL by both the methods. Overall incidence of ESBL production in uropathogens is less $(21.05 \%)$ in our study which is comparable with the study of Lee $\mathrm{D}$ et al ${ }^{26}$ Tankhiwale et al have reported higher incidence of ESBL production among urinary isolates ${ }^{5}$

ESBL producing strains are resistant to a wide variety of commonly used antimicrobials. Their proliferation possesses a serious global health concern that has complicated strategies for a growing number of hospitalized patients. Irrational prescription of antimicrobials, available over the counter in India, has contributed to this situation. Hence routine ESBL testing for uropathogens along with conventional antibiogram would be useful for all cases of UTI.

\section{REFERENCES:}

1. Pfaller MA, Segreti j. overview of the epidemiological profile and laboratory detection of extended spectrum beta lactamases.Clin infect Dis 2006;42;s153-s163.

2. Ruiz J, Simon K, Horcajada J, Velasco M, Barranco M, Roig G, Martinez A, Martinez J, Jimenez de Anta T, Mensa J, Vila J. Differences in virulence factors among clinical isolates of Escherichia coli causing cystitis and pyelonephritis in women and prostatitis in men. J.Clin.Microbiol. 2002; 40: 4445-4449.

3. Kaper JB, Nataro JP, Mobley HL. Pathogenic Escherichia coli. Nat Rev Microbiol. 2004; 2: 123-140.

4. Wullt B. The role of P fimbriae for Escherichia coli establishment and mucosal inflammation in the human urinary tract. Int J Antimicrob Agents. 2002; 21: 605-621.

5. Tankhiwale SS, Jalgoankar SV, Ahamad S, Hassani U. Evaluation of extended spectrum beta lactamase in urinary isolates. Indian J Med Res. 2004; 120: 553-556.

6. Chaudhary U, Aggarwal R. Extended spectrum $\beta$ - lactamases (ESBLs)- An emerging threat to clinical therapeutics. Indian J Med Microbiol. 2004; 22: 75-80.

7. Menon T, Bindu D, Kumar CPJ, Nalini S, Thirunarayan MA. Comparison of double disc and three dimensional methods to screen for ESBL producers in a tertiary care hospital. Indian J Med Microbiol. 2006; 24: 117-120.

8. Grubenberg GN. Antibiotic sensitivities of urinary pathogens: 1971-1982. J Antimicrob Chemother. 1984; 14: 17-23.

9. Jones RN, Thornsberry C. Cefotaxime: a review of in vitro antimicrobial properties and spectrum of activity. Rev Inf Dis. 1982; 4: 5300-15.

10. Fu KP, Neu HC. Betalactamase stability of HR 756 a novel cephalosporin, compared to that of cefuroxime and cefotaxime. Antimicrobials Agents Chemother. 1978; 14: 322326.

11. Nokashino SS, Nakamuro M. In vitro activity of cefotaxime against clinically significant pathogens. Drugs. 1988; 35(2): 14-21.

12. Beckford Ball J. Related articles, management of suspected bacterial urinary tract infection. Nurs Times. 2006; 102(36): 25-6. 
13. Girou E, Rioux C, Brun- Buisson C, Lobel B: Infection committee of the French Association of Urology. The postoperative bacteriuria score: a new way to predict nosocomial infection after prostate surgery. Infect Control Hosp Epidemiol. 2006; 27(8): 847-54.

14. Crichton PB. Enterobacteriaceae: Escherichia, Klebsiella, Proteus and other genera. Chapter 20. In: Mackie and McCartney Practical Medical Microbiology. 14th ed. Collee JG, in: Fraser AG, Marmion BP, Simmons A, Eds. ( Churchill Livingstone, New York ) 1996.p. 361-384.

15. Forbes BA, Sahm DF, Weissfeld AS. (Eds) Enterobacteriaceae. Chapter 25. In: Bailey and Scott's Diagnostic Microbiology. 11 th ed. Mosby: St.Louis; 2002.p. 365-377.

16. Shukla,I, Tiwari R,Agrawal $M$ prevalence of extended spectrum beta lactamase producing klebsiella pnuemoniae in a tertiary care hospital.IJMM 2004 April ;22(2) 8791.

17. Clinical and Laboratory Standard institute: Performance standards for antimicrobial susceptibility testing .nineteenth International supplement m100-s20. Clinical Laboratory Standards institute. Wayne, $\mathrm{Pa}(2010)$.

18. Varma NC, Taneja OP, Saxena SN. Recurrent urinary tract infections in females. J Ind Med Ass. 1972; 58: 155-58.

19. Gupta V, Yadav A, Joshis RM. Antibiotic resistance patterns in uropathogens. Indian J Med Microbiol. 2002; 20(2): 96-98.

20. Bajaj JK, Karyakarte RP, Kulkarni JD, Deshmukh AB. Changing etiology of urinary tract infections and emergence of drugs resistance as a major problem. J Commun Dis. 1999; 31(3): 181-84.

21. Johnson JR. Urinary tract infection. In: Sussman M, editor Escherichia coli: mechanisms of virulence, $1^{\text {st }}$ ed Cambridge: Cambridge University Press; 1997 p.495-549.

22. Kunin CM. The Concept of "significant bacteriuria" and asymptomatic bacteriuria, clinical syndromes and the epidemiology of urinary tract infections. In: Detection, prevention and management of urinary tract infections, $4^{\text {th }}$ ed. Philadelphia: Lea and Fiebiger; 1987. 57-124.

23. Moland ES and K.S. Thompson. Extended spectrum b-lactamases of Enterobactericeae. J Antimicrob. Chemother. 1994; 33:666-668.

24. Akata F, Tatman-Otkum M, Ozkan E, Tansel 0, Otkum M, Tugrul M. Prevalence of extended spectrum beta lactamases produced by nosocomial isolates of enterobacteriaceae in Trakta University Hospital, Turkey. New Microbiol. 2003; 26: 257 62.

25. Iqbal M, Patel IK, Shal SH, Ain Q, Barrey N, Kiani Q, et al. Susceptibility patterns of Escherichia coli prevalence of multidrug resistant isolates and extended spectrum beta lactamase phenotype. J Pak Med Assoc. 2002; 52: 407-11.

26. Lee DS, Lee CM, Lee SJ. Prevalence and risk factors for extended spectrum beta lactamase producing uropathogens in patients with urinary tract infection. Korean J Urol. 2010; 51: 492-497. 


\section{OBSERVATION TABLES}

Table No. 1 Bacterial isolates in symptomatic UTI cases

\begin{tabular}{|l|l|l|l|}
\hline S.No & Bacterial isolates & No. & Percentage \\
\hline 1 & E. coli & 202 & 57.7 \\
\hline 2 & Klebsiella pneumoniae & 99 & 28.3 \\
\hline 3 & Pseudomonas aeruginosa & 30 & 8.6 \\
\hline 4 & Proteus mirabilis & 08 & 2.3 \\
\hline 5 & Acinetobacter spp & 02 & 0.57 \\
\hline 6 & Staphylococcus aureus & 01 & 0.28 \\
\hline 7 & Enterococcus faecalis & 08 & 2.3 \\
\hline & Total & 350 & \\
\hline
\end{tabular}

Table No.2 Age and sex distribution of patients with culture proven UTI

\begin{tabular}{|l|l|l|}
\hline Age in years & Male & Female \\
\hline $0-10$ & $03(2.17 \%)$ & $08(3.77 \%)$ \\
\hline $11-20$ & $07(5.07 \%)$ & $28(13.20 \%)$ \\
\hline $21-30$ & $19(13.76 \%)$ & $89(41.98 \%)$ \\
\hline $31-40$ & $16(11.59 \%)$ & $33(15.56 \%)$ \\
\hline $41-50$ & $62(44.92 \%)$ & $28(13.20 \%)$ \\
\hline$>50$ & $31(22.46 \%)$ & $26(12.26 \%)$ \\
\hline Total & 138 & 212 \\
\hline
\end{tabular}

Table 3: Antibiotic resistant pattern of gram negative urinary isolates from symptomatic UTI cases

\begin{tabular}{|l|l|l|l|l|l|l|l|l|l|l|}
\hline Antibiotics & \multicolumn{2}{l|}{ E. coli } & \multicolumn{2}{l|}{ K.pneumoniae } & \multicolumn{2}{l|}{ Ps.aeruginosa } & \multicolumn{2}{l|}{ Pr.mirabilis } & \multicolumn{2}{l|}{$\begin{array}{l}\text { Acinitobacter } \\
\text { spp }\end{array}$} \\
\cline { 2 - 13 } & $\begin{array}{l}\text { OPD } \\
(74)\end{array}$ & $\begin{array}{l}\text { IPD } \\
(128)\end{array}$ & $\begin{array}{l}\text { OPD } \\
(14)\end{array}$ & $\begin{array}{l}\text { IPD } \\
(85)\end{array}$ & $\begin{array}{l}\text { OPD } \\
(04)\end{array}$ & $\begin{array}{l}\text { IPD } \\
(26)\end{array}$ & $\begin{array}{l}\text { OPD } \\
(02)\end{array}$ & $\begin{array}{l}\text { IPD } \\
(06)\end{array}$ & $\begin{array}{l}\text { OPD- } \\
\text { IPD } \\
(02)\end{array}$ \\
\hline Ampicillin & 52 & 120 & 09 & 62 & ND & ND & - & 03 & - & 02 \\
\hline Cephalexin & 36 & 74 & 06 & 58 & ND & ND & - & 04 & - & 02 \\
\hline cefotaxime & 29 & 69 & 06 & 52 & - & 13 & - & - & - & 01 \\
\hline Ceftazidime & 31 & 67 & 10 & 48 & 01 & 11 & - & 01 & - & 01 \\
\hline Amikacin & 24 & 52 & 04 & 41 & - & 04 & 01 & 01 & - & 01 \\
\hline Gentamicin & 36 & 52 & 04 & 40 & 02 & 14 & - & 03 & - & 01 \\
\hline Nitrofurantoin & 19 & 05 & 05 & 45 & ND & ND & - & 04 & - & 02 \\
\hline Nalidixic Acid & 37 & 72 & 06 & 47 & ND & ND & 01 & 03 & - & 02 \\
\hline Norfloxacin & 34 & 69 & 05 & 43 & 01 & 07 & - & 01 & - & 01 \\
\hline Cotrimoxazole & 29 & 64 & 07 & 39 & ND & ND & 01 & 04 & - & ND \\
\hline Piperacillin & ND & ND & ND & ND & - & 03 & ND & ND & - & ND \\
\hline
\end{tabular}

Figures in parenthesis indicate number of isolates $\quad *$ ND-Not detected 
Table No.4 Comparison of Modified DDST and PCT for ESBL detection in gram negative isolates.

\begin{tabular}{|c|c|c|c|c|c|c|c|}
\hline \multirow{2}{*}{ Bacterial isolates } & \multirow{2}{*}{$\begin{array}{l}\text { No of isolates } \\
\text { resistant to } 3^{\text {rd }} \\
\text { generation } \\
\text { cephalosporins }\end{array}$} & \multicolumn{3}{|c|}{ Modified DDST } & \multicolumn{3}{|l|}{ PCT } \\
\hline & & OPD & IPD & Total & OPD & IPD & Total \\
\hline E. coli & 98 & 05 & 09 & 14 & 06 & 13 & 19 \\
\hline K. pneumoniae & 58 & 03 & 09 & 12 & 03 & 13 & 16 \\
\hline Ps. aeruginosa & 13 & - & 01 & 01 & - & 01 & 01 \\
\hline Pr.mirabilis & 01 & - & - & - & - & - & - \\
\hline Acinetobacter spp & 01 & - & - & - & - & - & - \\
\hline Total & & & & $\begin{array}{l}27 \\
(15.78 \%)\end{array}$ & & & $\begin{array}{l}36 \\
(21.05 \%)\end{array}$ \\
\hline
\end{tabular}

